\title{
The intestinal microbial community dissimilarity in hepatitis B virus-related liver cirrhosis patients with and without at alcohol consumption
}

\author{
Yong-Dong Deng ${ }^{1}$, Xue-Bin Peng ${ }^{1}$, Rong-Rong Zhao ${ }^{1}$, Chao-Qun Ma², Jian-ning Li ${ }^{1}$ and Li-Qiong Yao ${ }^{3 *}$
}

\begin{abstract}
Background: Chronic hepatitis B virus (HBV) infection-reduced liver functions are associated with intestinal microbial community dissimilarity. This study aimed to investigate the microbial community dissimilarity in patients with different grades of HBV-related liver cirrhosis.

Results: Serum endotoxin was increased with Child-Pugh (CP) class (A, B, and C). Veillonellaceae and Lachnospiraceae families were reduced in patients compared with controls. Megamonas and Veillonella genus was reduced and increased in patients compared with controls, respectively, especially in CPB and CPC groups. Correlation analysis showed that endotoxin content was significantly correlated with alcohol consumption (95\% Cl 0.100, 0.493), CP class $(95 \% \mathrm{Cl} 0.289,0.687)$ and Lachnospiraceae family level $(95 \% \mathrm{Cl}-0.539,-0.122)$. Firmicutes/Bacteroidetes ratio was correlated with the level of Lachnospiraceae family (95\% Cl 0.013, 0.481), Veillonellaceae family $(95 \% \mathrm{Cl} 0.284,0.696)$, Megamonas genus $(95 \% \mathrm{Cl} 0.101,0.518)$ and Veillonella genus $(95 \% \mathrm{Cl} 0.134,0.545)$. All aforementioned bacteria were independent risk or protective factors for hepatitis. Alcohol consumption changed microbial community.
\end{abstract}

Conclusions: Our study demonstrated that elevated Firmicutes/Bacteroidetes ratio, reduced Megamonas genus level and increased Veillonella genus level were indicators for HBV-related liver cirrhosis. Alcohol-related pathogenesis was associated with the changed microbial community.

Keywords: Hepatitis B virus-related liver cirrhosis, Intestinal microbiota, 165 rRNA gene sequencing, Child-Pugh class

\section{Highlights}

1. Serum endotoxin increased in patients with higher Child-Pugh class.

2. Alcohol consumption increased endotoxin in ChildPugh class A patients.

3. Megamonas and Veillonella was reduced and increased in patients vs. control, respectively.

4. Alcohol consumption changed microbial community in patients.

\footnotetext{
*Correspondence: yliq20032003@163.com

${ }^{3}$ Department of Laboratory, First Hospital of Lanzhou University, \#1

Donggang West Road, Lanzhou 730000, Gansu, China

Full list of author information is available at the end of the article
}

5. Firmicutes/Bacteroidetes ratio increment correlated with hepatitis pathogenesis.

\section{Background}

Hepatitis B virus (HBV) infection is a global public health problem. The prevalence of chronic HBV infection ranges from 0.20 to $22 \%$ in different countries [1-3]. There are increasing evidence showing the relevance of intestinal microbiota with hepatosis, like HBV-related liver cirrhosis $[4,5]$.

Chronic HBV infection reduces liver functions and eventually gives rise to liver cirrhosis, hepatitis and hepatocellular carcinoma (HCC). The significant dissimilarities in intestinal microbial community between 
chronic hepatitis B or HBV-related liver cirrhosis and healthy controls have been widely reported $[4,6]$. With comparison to healthy controls, patients with chronic hepatitis B showed abundant Anaerostipes taxon [7]. Liu et al. reported that patients with HBV-related HCC had reduced levels of pro-inflammatory bacteria like Escherichia-Shigella and increased level of anti-inflammatory bacteria like Faecalibacterium, while patients with nonHBV and non-hepatitis C virus (HCV) HCC showed opposite results [8]. Additionally, the altered abundance of gut microbial community has been identified in nonalcoholic fatty liver disease (NAFLD) and is deemed to be associated with progression into non-alcoholic steatohepatitis (NASH) [5, 9]. However, alcohol consumption itself induces injury and inflammation in the intestine and liver, and dissimilarity in microbial community [10, 11]. For instance, Stearns et al. showed that alcohol consumption increased the ratio of Firmicutes to Bacteroidetes [11], which is used as a parameter of obese [12, 13], lipid metabolism [14] and insulin resistance [15]. These observations showed gut microbiota dysbiosis was relevant to HBV infection and might benefit in the progression to severe liver failure like HCC and liver disease. However, the association of microbial community dissimilarity with patients with HBV-related liver cirrhosis was unclear till now.

We performed this study to investigate the microbial community dissimilarity in healthy and patients with different grades of HBV-related liver cirrhosis. The present study included a study cohort of 80 patients with HBVrelated liver cirrhosis at Child-Pugh class A, B, C and 20 healthy controls without known liver diseases. The microbial community diversity in different groups were analyzed and compared. And alcohol history of each patient was asked for the analysis of alcohol consumption-related factors. The bacteria associated with hepatitis were identified. According to the reported relation of Firmicutes/Bacteroidetes ratio to diseases [15], we also performed logistics analysis to identify the correlation of this ratio with disease progression.

\section{Methods}

\section{Patient population}

Before experiments, an ethical approval and consent to participate (LDYYLL2018-142) was obtained from the ethical committees of First Hospital of Lanzhou University, Lanzhou, China. This study enrolled 80 patients (han Chinese) with HBV-related cirrhosis (including 55 males and 25 females, aged $42.30 \pm 13.15$ years) admitted in our hospital from July 2018 to Dec 2018 . All patients with HBV-related cirrhosis were staged according to the Child-Pugh (CP) class A (CPA, $\mathrm{n}=30), B(\mathrm{CPB}, \mathrm{n}=31)$ and $\mathrm{C}(\mathrm{CPC}, \mathrm{n}=19)$. All patients did not receive probiotics and antibiotics for 8 weeks prior to admission. Patients with solid organ transplantation, HCC, drug-induced liver injury, autoimmune liver disease and alcoholic fatty liver were excluded. In addition, patients with specific food habits, like vegetarians and lactose lovers, were excluded from our study. Twenty healthy volunteers (age- and sex-matched) without known diseases were randomly selected from the physical examination center of our hospital. Use of antibiotics within 8 weeks prior sample collection was forbidden. Alcohol history and consumption (white liquor) of all patients was asked. Fasting blood and stool samples were collected from all participants with informed consent. All samples were set at $-80{ }^{\circ} \mathrm{C}$ before examination. This study was performed according to the Declaration of Helsinki (1975).

\section{Blood biochemical indicator detection}

The serum diamine oxidase (DAO), D-lactate, endotoxin and HBV-DNA was detected using highly sensitive human DAO ELISA kit, lactic acid and bacterial endotoxin assay kits (JY-Po-Color DLT Set; Zhongsheng jinyu diagnostic technology co. LTD., Beijing, China), respectively. Three duplicates were setup for each sample.

\section{DNA extraction and sample preparation}

Total DNA samples were extracted from stool samples using MoBioPowersoil DNA extraction kits (MoBio, Carlsbad, CA, USA) following the manufacturer's instructions. DNA quality was determined using $1 \%$ agarose gel electrophoresis. Amplification of the V4 region of the 16S rRNA gene was conducted using the 515 (Forward, 5'-GTGCCAGCMGCCGCGGTAA-3')/806 (Reverse, 5'-GGACTACHVGGGTWTCTAAT-3') primers and TransGen AP221-02: TransStartFastpfu DNA Polymerase (TransGen Biotech, Beijing, China). Amplification of $20 \mu \mathrm{L}$ reactions was implemented on Applied Biosystems GeneAmp ${ }^{\circledR} 9700$ (Applied Biosystems, Foster City, CA, USA) at $94{ }^{\circ} \mathrm{C}$ for $4 \mathrm{~min}$, followed by 30 cycles of $94{ }^{\circ} \mathrm{C}$ for $40 \mathrm{~s}, 58{ }^{\circ} \mathrm{C}$ for $30 \mathrm{~s}$, and $72{ }^{\circ} \mathrm{C}$ for $45 \mathrm{~s}$, and followed by a final extension at $72{ }^{\circ} \mathrm{C}$ for $10 \mathrm{~min}$. Three duplicates were set up for each sample. PCR products from one sample were pooled and then gel purified ( $2 \%$ agarose gel) using an AxyPrep DNA Gel Extraction kit (Axygen Biosciences, Hangzhou, China). DNA quantification was performed using QuantiFluor ${ }^{\mathrm{TM}}$ (Promega, Lyon, France). DNA library construction was carried out using the TruSeq ${ }^{\circledR}$ DNA PCR-Free Sample Preparation Kit (Illumina, San Diego, USA) and 16S rRNA gene sequencing was conducted on the Illumina MiSeq platform (Illumina, San Diego, USA; $2 \times 250 \mathrm{bp} \mathrm{PE}$ ). 


\section{Data processing and bioinformatics analysis}

Raw reads were obtained and merged using FLASH (version 1.2.7; http://ccb.jhu.edu/software/FLASH/). Lowquality bases were trimmed using the Trimmomatic program (version 0.36, http://www.sadellab.org/cms/ index.php?page=trimmomatic). The sorting of microbial operational taxonomic units (OTUs) and taxonomic assignments was performed using Qiime (v1.9.1; http:// qiime.org/scripts/split_libraries_fastq.html). Chimera sequences in OTUs were removed using Usearch (version 7.1, http://drive5.com/uparse/). Annotation of OTUs was performed in SILVA's SSU rRNA database (http:// www.arb-silva.de/). The OTUs' alpha diversity estimators, including community richness (Chao1), community diversity (Shannon and Simpson indices) and sequencing depth (Good's coverage) were analyzed using Mothur (version v.1.30.1, http://www.mothur.org/wiki/Schloss SOP\#Alpha_diversity). Rarefaction curves were analyzed using Mothur. OTUs with at $97 \%$ identity and $>1 \%$ relative abundance were retained for further analysis. Beta diversities (Bray-Curtis dissimilarity) between samples were analyzed using Qiime. Principal co-ordinates analysis (PCoA; Bray-Curtis distance) was performed using $\mathrm{R}$ programing language.

\section{Statistical analyses}

All clinical data were expressed as mean \pm standard deviation or median and range $(\mathrm{Q} 1-\mathrm{Q} 3)$ and were analyzed using SPSS 22.0. Comparisons in blood biochemical indicators were performed using non-parametric Mann-Whitney U test, Kruskal-Wallis $H$ test or $\chi^{2}$ test. Differences between alcohol and non-alcohol consumed patients were analyzed using Mann-Whitney U test. For gut microbiota analysis, non-parametric KruskalWallis $\mathrm{H}$ test and Wilcoxon rank-sum test was used for the differences in alpha and beta diversities. Differences in taxonomies were analyzed using non-parametric Kruskal-Wallis sum-rank test and LDA Effect Size
(LEfSe) software (http://huttenhower.sph.harvard.edu/ galaxy/root?tool_id=lefse_upload). Differences in the relative abundances of OTUs of dominant bacteria were analyzed using Welch's $t$ test or Mann-Whitney $U$ test. Spearman correlation coefficients ( $r$ ) between dominant bacteria and blood biochemical indicators were identified. Logistic regression analysis was performed to identify the indicators for disease progression. $\mathrm{p}<0.05$ was considered statistically significant.

\section{Results}

\section{Differences in serum indicators}

There was no difference in the age, male ratio, and alcohol consumption frequency among the three groups (Table 1). The difference in the content of serum DAO (range 0.86-14.20 U/L), D-lactate (2.46-20.75 mg/L) and endotoxin (0.12-23.59 U/L) are shown in Table 1. Serum $\mathrm{DAO}$ and D-lactate contents were equivalent in patients with CP class A $(n=30), B(n=31)$ and $C(n=19)$ HBVrelated cirrhosis. Serum endotoxin and HBV-DNA contents were increased in patients with higher $\mathrm{CP}$ classes $(\mathrm{p}=0.000$ and $\mathrm{p}=0.000$, respectively; Table 1$)$. Alcohol consumption increased the serum endotoxin in all patients $(\mathrm{p}=0.006$, Table 2$)$; endotoxin and D-lactate contents in CPA patients $(\mathrm{p}=0.008$ and 0.017 , respectively); but decreased DAO content in CPC patients $(\mathrm{p}=0.035)$.

\section{Sequencing depth estimation}

Illumina sequencing generated 4,661,253 sequences (ranging 251-300 bp), with an average length of $255.76 \mathrm{bp}$. These sequences represented 4414 OTUs with $97 \%$ identity after data processing. Rarefaction curves and Shannon-Wiener curves reached plateaus, indicating sufficient sequencing depths for microbial diversity analysis (Fig. 1a, b). Venn diagram showed there were 1724 overlapped OTUs (Fig. 1c). PCoA based on the

Table 1 Demographical characteristics and blood biochemical indicators in patients

\begin{tabular}{|c|c|c|c|c|}
\hline Indicators & $C P A^{a}$ & $C P B^{b}$ & $\mathrm{CPC}^{\mathrm{C}}$ & p \\
\hline Patients & 30 & 31 & 19 & \\
\hline Age (years) & $46.5(34-67)$ & $48.7(32-67)$ & $52.8(38-57)$ & $0.571^{*}$ \\
\hline Gender (male) & $66.67 \%(20 / 30)$ & $63.33 \%(19 / 31)$ & $68.42 \%(13 / 19)$ & $0.851^{@}$ \\
\hline Alcohol consumption (Yes/no) & $23.33 \%(7 / 30)$ & $48.38 \%(15 / 31)$ & $47.37 \%(9 / 19)$ & $0.114^{@}$ \\
\hline $\mathrm{DAO}(\mathrm{U} / \mathrm{L})$ & $2.26(1.88-2.61)$ & $2.37(1.85-2.72)$ & $2.39(1.54-2.98)$ & $0.799 *$ \\
\hline D-lactate (mg/L) & $10.29(8.35-12.83)$ & $8.53(7.20-12.78)$ & $11.27(6.320-14.260)$ & $0.570^{*}$ \\
\hline LPS (U/L) & $4.70(2.88-8.26)$ & $7.26(5.72-9.66)$ & $11.64(9.970-17.000)$ & $0.000^{*}$ \\
\hline HBV-DNA & 0 & $0\left(0-1.05 \times 10^{4}\right)$ & $1.302 \times 10^{6}\left(0-5.510 \times 10^{6}\right)$ & $0.000^{*}$ \\
\hline
\end{tabular}

*and ${ }^{\circledR}$, differences were analyzed using non-parametric Kruskal-Wallis $\mathrm{H}$ test and $\mathrm{X}^{2}$ test, respectively

$a, b, c$ Patients with Child-Pugh class A $(n=30), B(n=31)$ and C $(n=19)$ hepatitis B or hepatitis B virus-related cirrhosis 


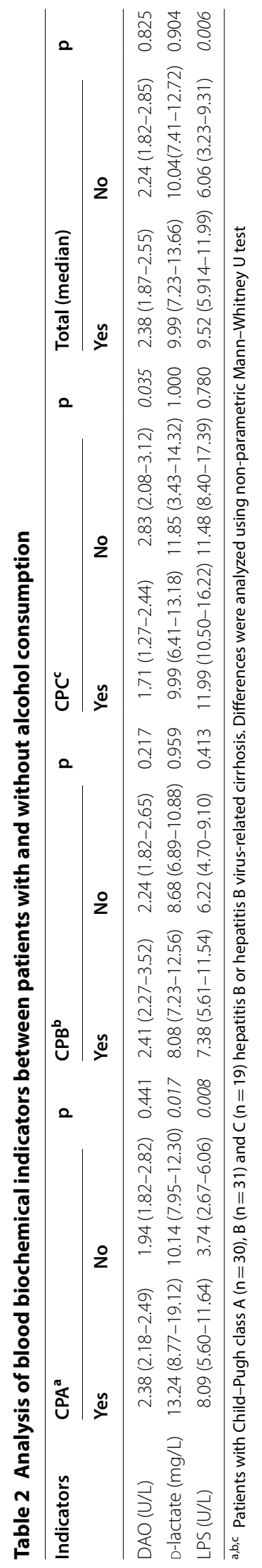



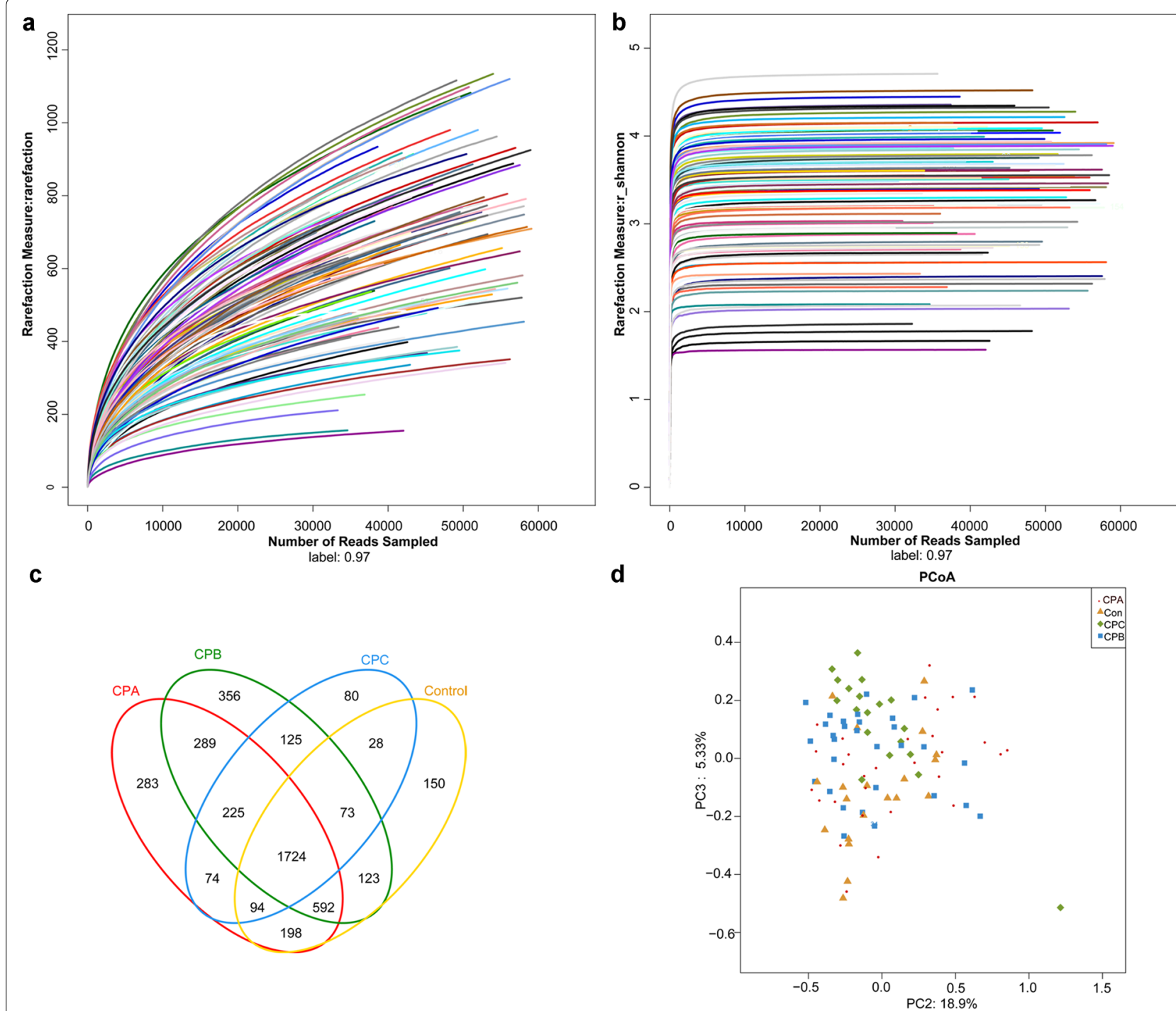

Fig. 1 Estimation of sequencing. a, b The rarefaction curves and Shannon-Wiener curves of samples, respectively. c The Venn diagram of the OTUs in groups. d The principal co-ordinates analysis (PCOA) plot of samples

Bray-Curtis distance showed there was no distinct clustering of species groups (Fig. 1d).

\section{Microbial community dissimilarity in patients}

Microbial diversity analysis showed that the relative abundances of Firmicutes and Bacteroidetes phylum in patients were equivalent with that in control (Fig. 2a). At the family level, Lachnospiraceae (Firmicutes phylum, $18.70 \pm 3.20 \%$ ) and Veillonellaceae (Firmicutes phylum, $14.73 \pm 3.13 \%)$ relative abundances in patients were significantly lower than that in controls $(27.64 \pm 2.17 \%$, $\mathrm{p}<0.0001$; and $23.16 \pm 3.73 \%, \mathrm{p}<0.0001$; Fig. $2 \mathrm{~b}, \mathrm{c})$. At the level of genus, Megamonas (Veillonellaceae family) relative abundance in patients $(5.14 \pm 1.17 \%)$ was significantly lower than that in controls $(14.80 \pm 4.67 \%$, $\mathrm{p}<0.0001$, Fig. 2d, e), and Veillonella genus showed opposed trends. Species taxonomy showed the dominant bacteria were uncultured bacteria of Faecalibacterium, Megamonas and Bacteroides. Patients had low abundance of an uncultured species of Megamonas (7.06 $\pm 2.74 \%$ ) compared with healthy controls $(14.77 \pm 3.39 \%$, $\mathrm{p}<0.0001$, Fig. 3a, b).

\section{Microbial communities in patients with different CP classes} We further performed the pairwise comparison between patients with different $\mathrm{CP}$ classes and controls in order to investigate the microbial community dissimilarity in patients with different disease severities. Microbial 

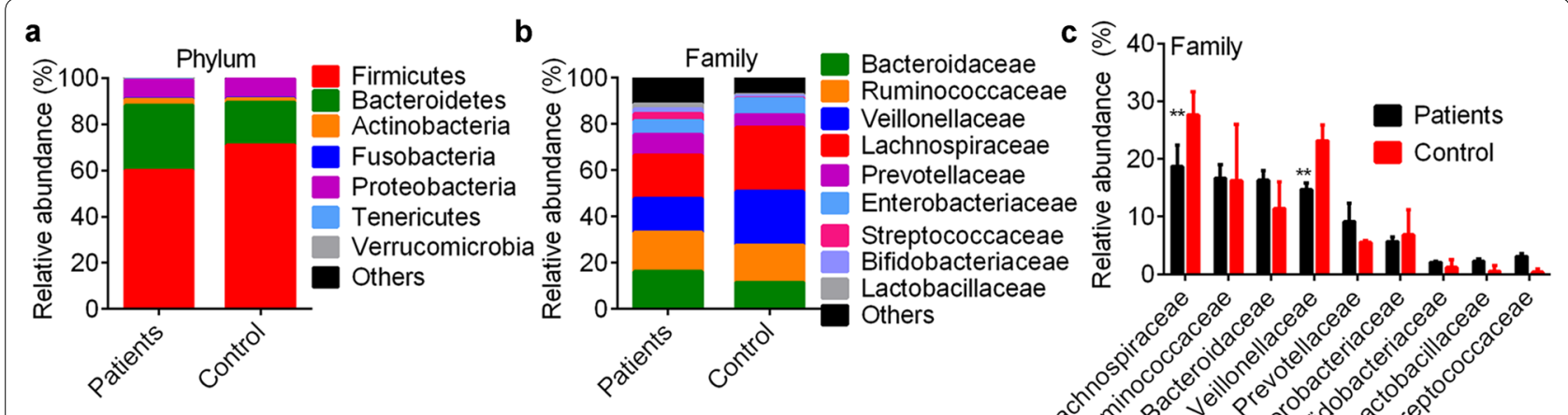

d

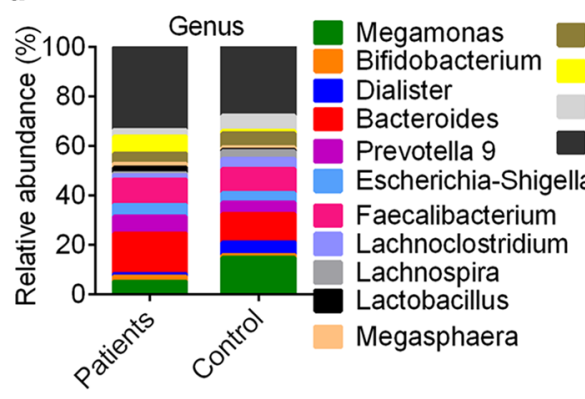

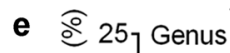
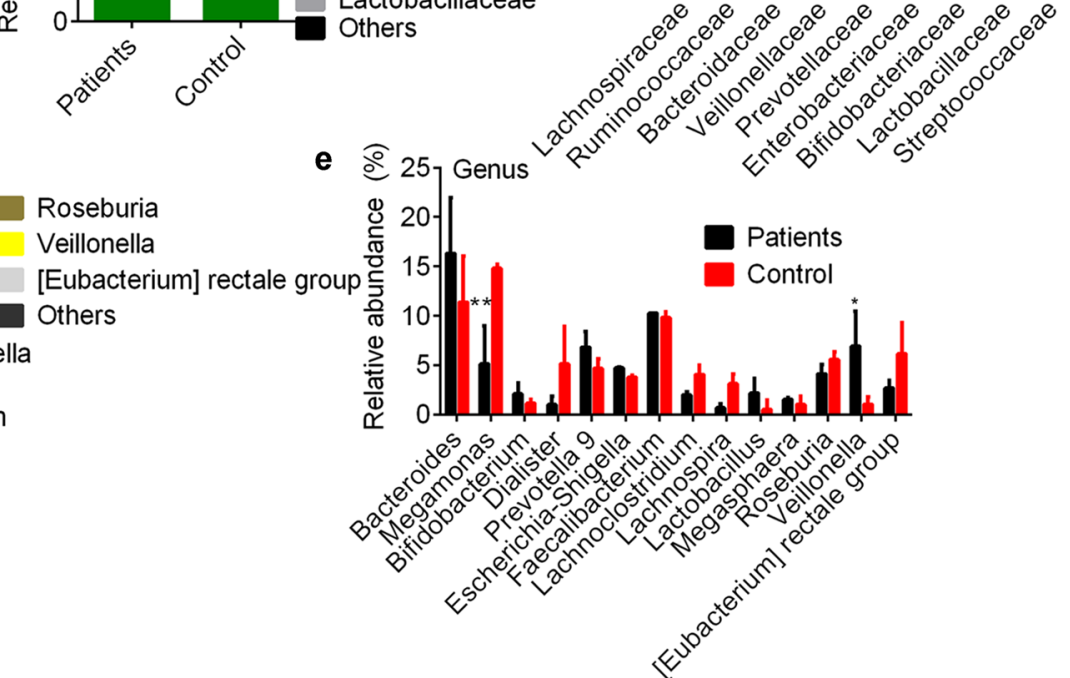

Fig. 2 Bacterial community in health controls and patients. Dominant bacterial abundance at the phylum level (a), family (b, c) and genus level (d, e). ${ }^{* *} \mathrm{q}<0.01$ vs. control by Wilcoxon rank-sum test

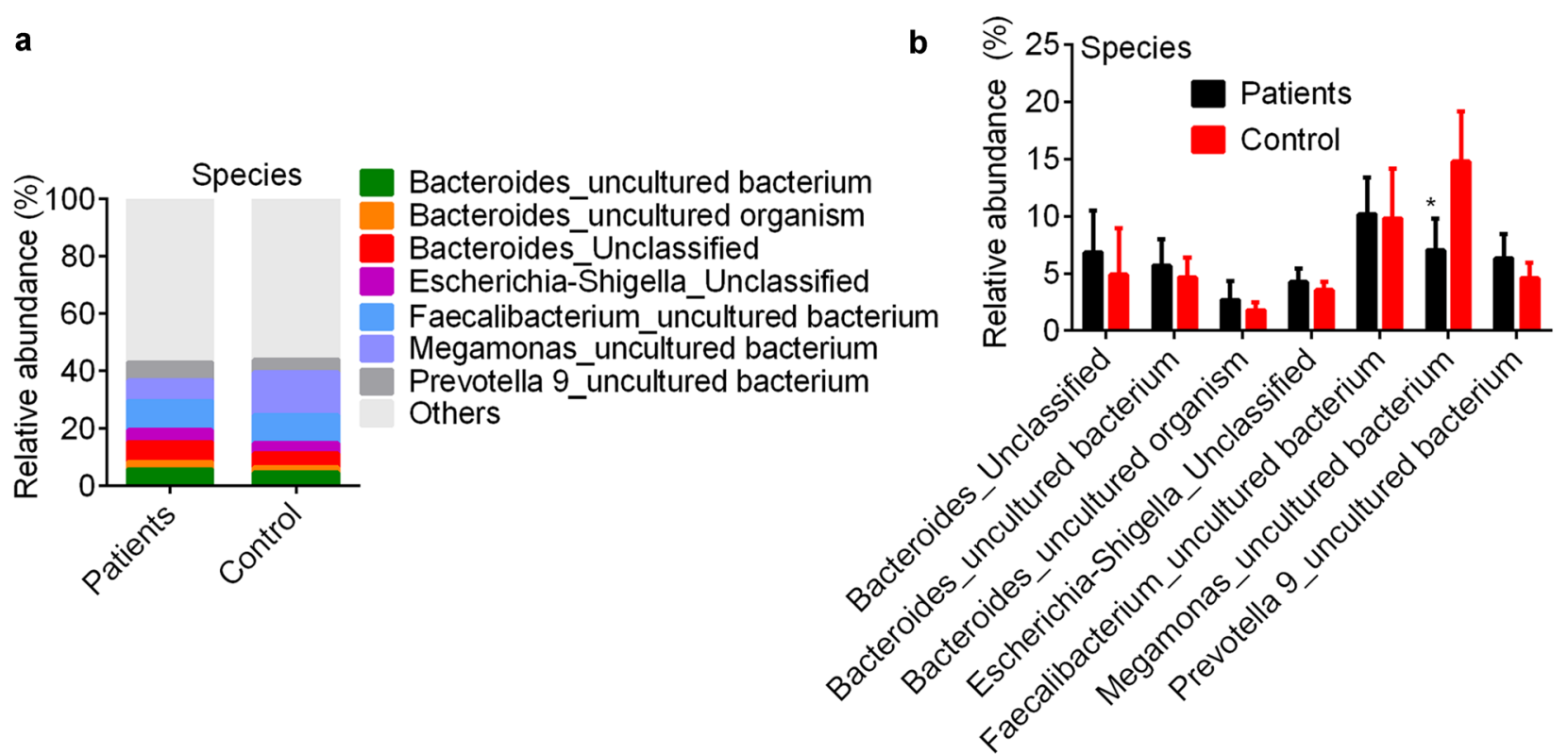

Fig. 3 a showes species abundance (> 1\%) between health controls and patients. b showes statistically significant effects of species abundance $(>1 \%)$ between groups. ${ }^{*} p<0.05$ vs. control by Wilcoxon rank-sum test 
community analysis showed there were no differences in the relative abundances of bacteria at the phylum (Fig. 4a) and family level (Fig. 4b, c). Lachnospiraceae (18.75-27.64\%), Ruminococcaceae (Firmicutes phylum, 16.02-19.07\%), Veillonellaceae (14.73-13.16\%) and Bacteroidaceae (Bacteroidetes phylum, 11.39-15.70\%) were dominant families in all groups. At the genus level, Faecalibacterium (9.71-10.82\%), Megamonas (1.61-14.80\%) and Bacteroides (11.39-16.33\%) were dominant taxonomies. Patients with $\mathrm{CPB}$ and $\mathrm{CPC}$ HBV-related liver cirrhosis had lower Megamonas level $(1.61 \pm 1.10 \%$ and $5.14 \pm 3.11 \%)$ and higher Veillonella level $(9.43 \pm 2.18 \%$ and $6.93 \pm 2.67 \%)$ compared with control $(14.30 \pm 5.11 \%$ and $1.05 \pm 0.84 \%$, respectively; $\mathrm{p}<0.0001$ for both) and patients with CPA disease $(11.95 \pm 2.31 \%$ and $2.41 \pm 1.55 \%$; $<<0.0001$, Fig. $4 d$, e). Species taxonomy dissimilarity analysis showed uncultured bacteria of Faecalibacterium, Megamonas and Bacteroides were dominant bacteria in all groups (Fig. 5a, b). The relative abundance of an uncultured species of Megamonas in patients with CPB $(1.61 \pm 0.74 \%)$ and CPC (5.13 $\pm 1.82 \%)$ disease was dramatically lower than that in controls $(14.77 \pm 3.39 \%$, $\mathrm{p}<0.0001)$ and patients with CPA disease $(11.93 \pm 2.92 \%$, $\mathrm{p}<0.0001$, Fig. 5b). LEfSe analysis showed that Mollicutes family (Tenericutes phylum), Lachnospiraceae family, Micrococcales family (Actinobacteria phylum) and Pasteurellales (Proteobacteria phylum) were important bacteria in CPA, CPB, CPC and control groups (Fig. 5c, d).

\section{Microbial community dissimilarity by alcohol consumption} In order to investigate the influence of alcohol consumption on microbial community dissimilarity, patients were divided into two groups: with and without alcohol consumption. There was no microbial community dissimilarity in the relative abundance of dominant phyla (data not shown), families (Fig. 6a) and genera (Fig. 6b) between the two groups. In patients within the same $\mathrm{CP}$ class, alcohol consumption induced significant microbial community dissimilarity at the family and genus levels (Fig. 6c, d). Alcohol consumption decreased Lachnospiraceae family $(11.41 \pm 3.68 \%$ vs. $25.37 \pm 8.03 \%$, $\mathrm{p}<0.0001)$ and Veillonellaceae family $(6.43 \pm 2.73 \%$ vs. $15.39 \pm 5.66 \%, \mathrm{p}<0.0001)$ in CPA patients; and increased Bacteriodaceae family in CPA patients $(23.37 \pm 4.67 \%$ vs. $12.94 \pm 5.13 \%, \mathrm{p}<0.0001$; Fig. $6 \mathrm{c}$ ). At the genus level, we found alcohol consumption increased Bacteroides (CPA and CPC) and Megamonas (CPA) and Veillonella (CPB) level, respectively (Fig. 6d). These differences

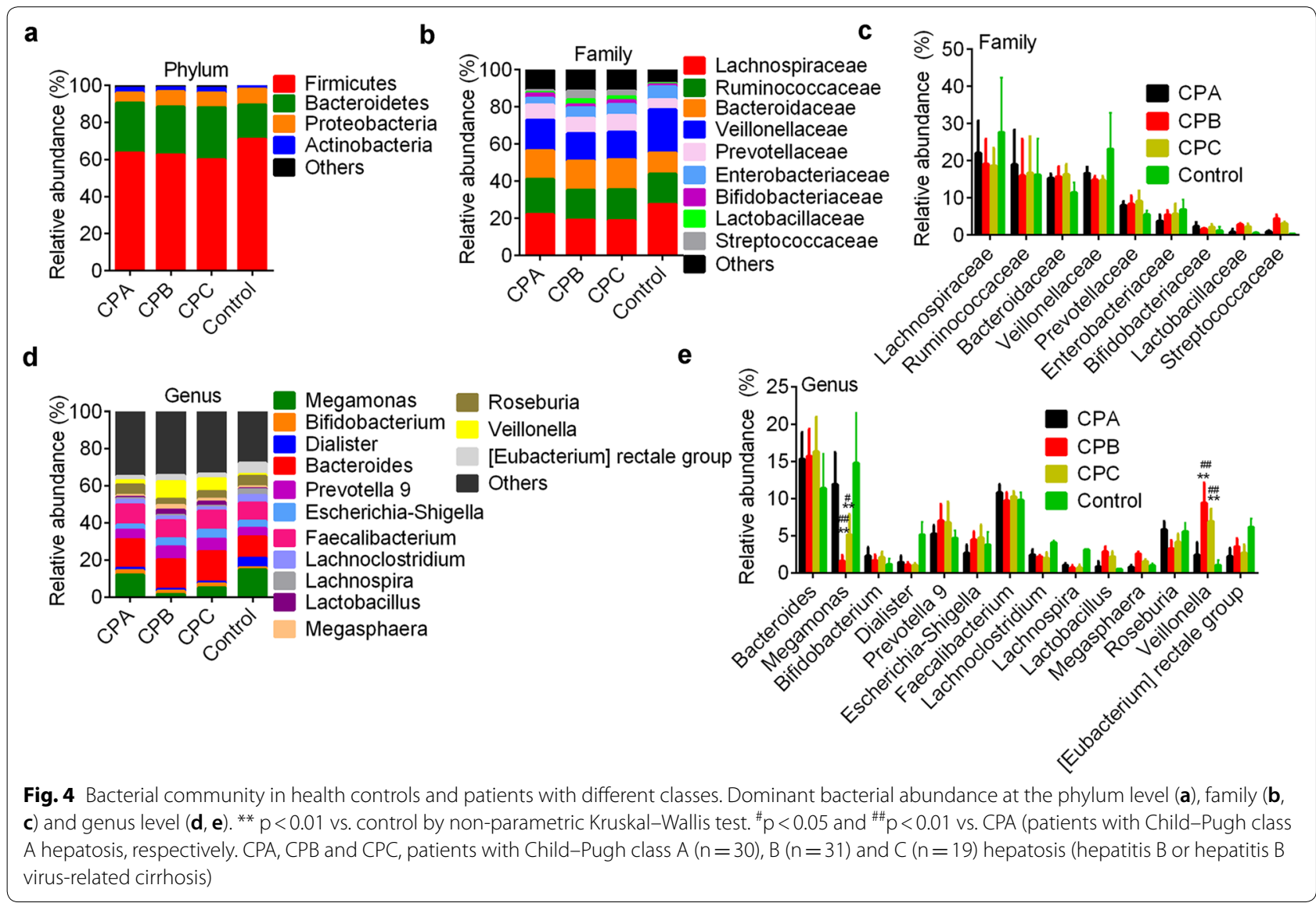




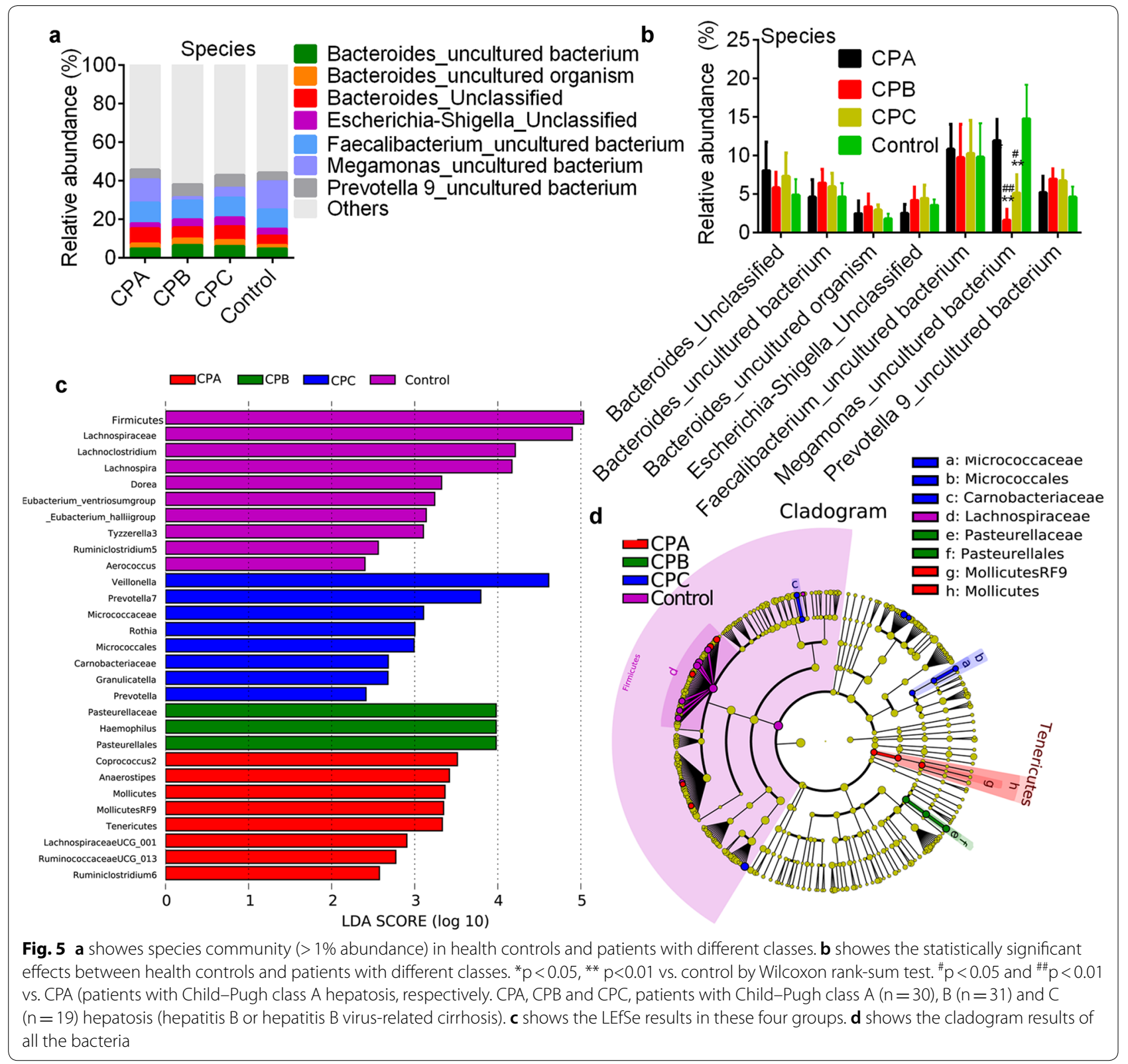

demonstrated that alcohol consumption caused microbial community dissimilarity in patients with HBVrelated cirrhosis.

\section{Correlation analysis for dominant bacteria}

To investigate the correlation of microbial community dissimilarity in patients with HBV-related liver cirrhosis, we performed Spearman correlation analysis. The results showed that endotoxin was correlated with alcohol consumption $(\mathrm{r}=0.314,95 \%$ CI $0.100,0.493, \mathrm{p}=0.006)$ and CP class $(\mathrm{r}=0.501,95 \%$ CI $0.289,0.687, \mathrm{p}=0.000)$. The relative abundance of Lachnospiraceae family was negatively correlated with endotoxin $(r=-0.345,95 \%$ CI $-0.539,-0.122, \mathrm{p}=0.002)$, HBV-DNA content $(\mathrm{r}=-0.315,95 \% \mathrm{CI}-0.497,-0.119, \mathrm{p}=0.006), \mathrm{CP}$ class $(\mathrm{r}=-0.410,95 \% \mathrm{CI}-0.589,-0.202, \mathrm{p}=0.001)$ and Bacteroidetes phylum level $(\mathrm{r}=-0.247,95 \% \mathrm{CI}$ - 0.453, 0.017, $\mathrm{p}=0.033$ ), while positively correlated with Firmicutes phylum level $(\mathrm{r}=0.395,95 \%$ CI 0.166 , $0.588, \mathrm{p}=0.000)$ and Firmicutes/Bacteroidetes ratio $(\mathrm{r}=0.278,95 \%$ CI $0.013,0.481, \mathrm{p}=0.016)$. Veillonella genus abundance was positively correlated with $\mathrm{CP}$ class $(\mathrm{r}=0.396,95 \%$ CI $0.211,0.573, \mathrm{p}=0.000)$, HBV-DNA content $(\mathrm{r}=0.403,95 \%$ CI $0.207,0.581, \mathrm{p}=0.000)$, Veillonellaceae family level $(\mathrm{r}=0.554,95 \%$ CI $0.313,0.761$, 


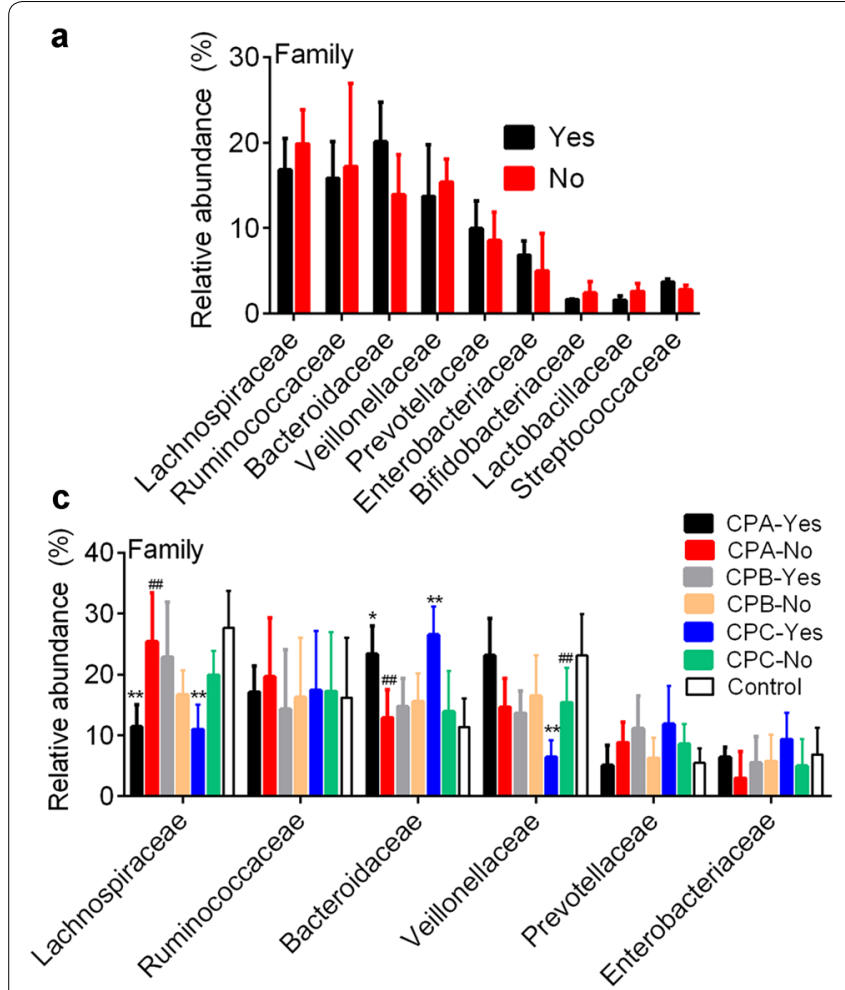

b
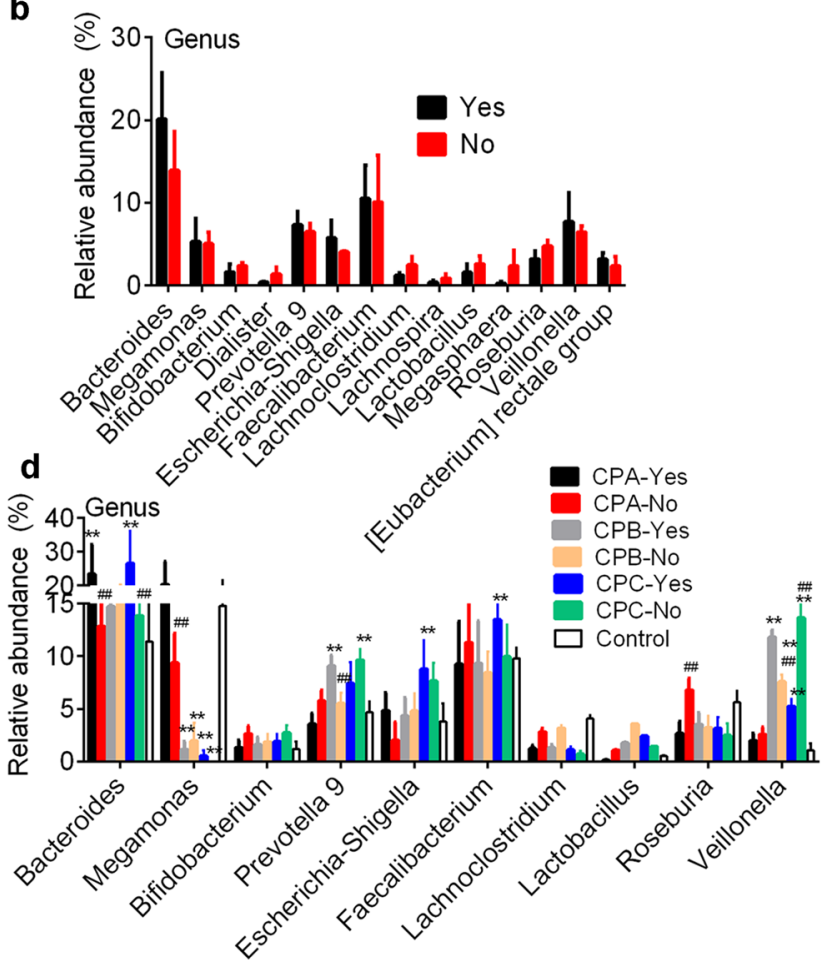

Fig. 6 a and $\mathbf{b}$ show the bacteria community dissimilarity in patients with (Yes) and without (No) alcohol consumption at family and genus level, respectively. $\mathbf{c}$ and $\mathbf{d}$ show the bacteria community dissimilarity in different groups with (Yes) and without (No) alcohol consumption at family and genus level, respectively. ${ }^{*} p<0.05$ and ${ }^{* *} p<0.01$ vs. control by Mann-Whitney test, respectively. ${ }^{\#} p<0.05$ and ${ }^{\#} p<0.01$ vs. patients without alcohol consumption (No), respectively. CPA, CPB and CPC, patients with Child-Pugh class $A(n=30), B(n=31)$ and C ( $n=19)$ hepatosis (hepatitis B or hepatitis $B$ virus-related cirrhosis)

$\mathrm{p}=0.000)$ and Firmicutes/Bacteroidetes ratio $(\mathrm{r}=0.356$, $95 \%$ CI 0.134, 0.545, $\mathrm{p}=0.000$; Table 3). Veillonella genus was negatively correlated with Bacteroidetes phylum level $(\mathrm{r}=-0.374,95 \% \mathrm{CI}-0.564,0.157, \mathrm{p}=0.001)$. The Firmicutes/Bacteroidetes ratio was positively correlated with Lachnospiraceae family level $(\mathrm{r}=0.278,95 \%$ CI 0.013 , $0.481, \mathrm{p}=0.016)$, Veillonellaceae family level $(\mathrm{r}=0.509$, 95\% CI 0.284, 0.696, $\mathrm{p}=0.000$ ), Megamonas genus level $(\mathrm{r}=0.321,95 \%$ CI 0.101, 0.518, $\mathrm{p}=0.005)$, Veillonella genus level $(\mathrm{r}=0.356,95 \% \mathrm{CI} 0.134,0.545, \mathrm{p}=0.002)$, and Firmicutes phylum level $(\mathrm{r}=0.876,95 \%$ CI $0.757,0.953$, $\mathrm{p}=0.000)$, while Bacteroidetes phylum $(\mathrm{r}=-0.985,95 \%$ CI $-0.995,-0.961, p=0.000$ ) was negatively correlated with these bacteria (Table 3). This was true for Firmicutes phylum.

Logistics analysis showed that the abundance of $\mathrm{Meg}$ amonas genus $\left(\beta=8.35295 \%\right.$ CI $1.863-9.467 \times 10^{6}$, $\mathrm{p}=0.034)$, Veillonella genus $(\beta=32.450,95 \% \mathrm{CI}$ $\left.2.298 \times 10^{4}, 6.673 \times 10^{23}, \mathrm{p}=0.005\right)$ and Firmicutes/Bacteroidetes ratio $(\beta=0.011,95 \%$ CI 1.002, $1.020, p=0.015)$ were an independent risk factors of hepatitis B development; while that of Lachnospiraceae family $(\beta=-8.518$,
95\% CI $0.000,0.406, \mathrm{p}=0.028$ ), Veillonellaceae family $(\beta=-15.311,95 \%$ CI $0.000,0.009, \mathrm{p}=0.005)$ were protective effect of this disease (Table 4). In patient with hepatitis $\mathrm{B}$, none of the aforementioned bacteria and blood biochemical diversities was significantly influenced by alcohol consumption (Table 5). Alcohol consumption showed borderline significance in elevating Megamonas genus level $\left(\mathrm{p}=0.055, \beta=35.693,0.476-2.129 \times 10^{31}\right.$, $\mathrm{p}=0.055)$. These results showed that alcohol consumption was not the independent risk factor for serum D-lactate, endotoxin, DAO and the microbial community dissimilarity in patients with HBV-related liver cirrhosis.

\section{Discussion}

Our present study demonstrated that intestinal microbial community diversity in patients with HBV-related liver cirrhosis was different from that in healthy controls. The intestinal microbial community dissimilarity, like reduced Megamonas genus (Veillonellaceae family) level, Lachnospiraceae family level as well as increased Veillonella genus (Veillonellaceae family) level, was associated with 


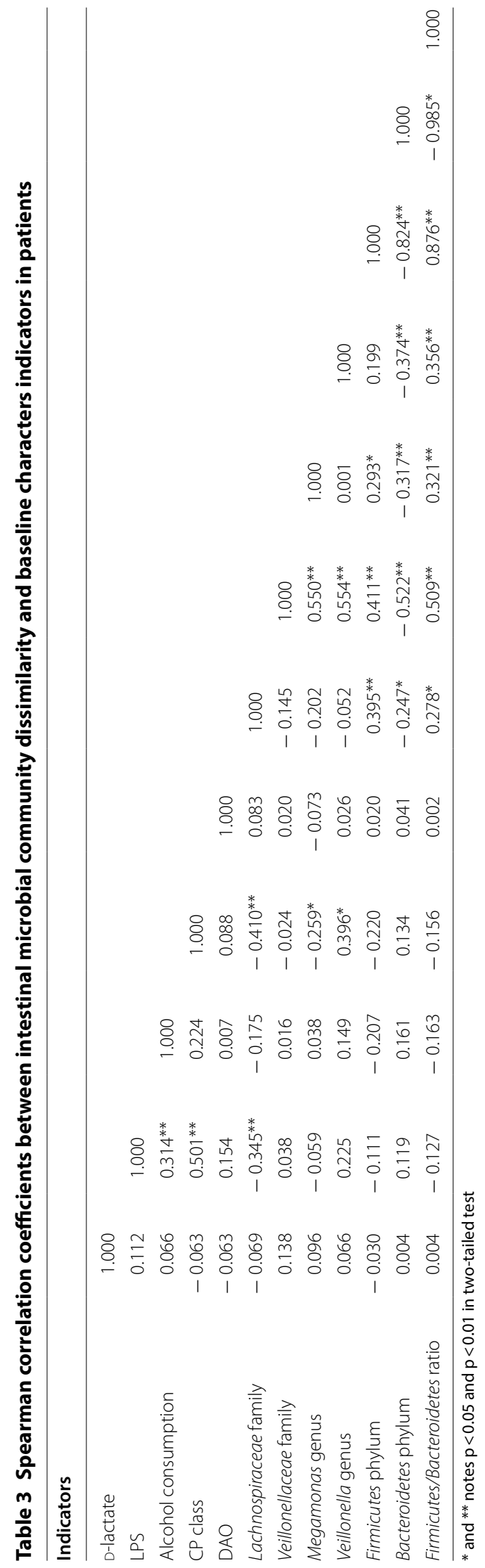


Table 4 The logistics analysis for the significantly changed bacteria associated with hepatitis

\begin{tabular}{lrll}
\hline Indicators & $\boldsymbol{\beta}$ & $\mathbf{9 5 \%} \mathbf{C l}$ & $\mathbf{p}$ \\
\hline Lachnospiraceae family & -8.518 & $0.000-0.406$ & 0.028 \\
Veillonellaceae family & -15.311 & $0.000-0.009$ & 0.005 \\
Megamonas genus & 8.352 & $1.863-9.467 \times 10^{6}$ & 0.034 \\
Veillonella genus & 32.450 & $2.298 \times 10^{4}$ to & 0.005 \\
& & $6.673 \times 10^{23}$ & \\
Firmicutes phylum & 7.507 & $0.804-4.119 \times 10^{6}$ & 0.057 \\
$\begin{array}{l}\text { Bacteroidetes phylum } \\
\text { Firmicutes/Bacteroidetes } \\
\text { ratio }\end{array}$ & 5.919 & $1.538-2.575 \times 10^{5}$ & 0.076 \\
\hline
\end{tabular}

Table 5 The logistics analysis for the indicators associated with alcohol consumption in patients

\begin{tabular}{lrll}
\hline Indicators & \multicolumn{1}{c}{$\boldsymbol{\beta}$} & $\mathbf{9 5 \%} \mathbf{C l}$ & $\mathbf{p}$ \\
\hline Lachnospiraceae family & 2.611 & $0.030-6.082 \times 10^{3}$ & 0.402 \\
Veillonellaceae family & -32.015 & $0.000-10.098$ & 0.079 \\
Megamonas genus & 35.693 & $0.476-2.129 \times 10^{31}$ & 0.055 \\
Veillonella genus & 36.621 & $0.135-4.753 \times 10^{32}$ & 0.063 \\
Firmicutes phylum & -2.917 & $0.000-20.298$ & 0.335 \\
Bacteroidetes phylum & 0.376 & $0.013-163.775$ & 0.876 \\
Firmicutes/Bacteroidetes ratio & -0.003 & $0.986-1.007$ & 0.522 \\
D-lactate & 0.059 & $0.931-1.209$ & 0.376 \\
LPS & 0.113 & $0.971-1.292$ & 0.119 \\
CP classes & 0.161 & $0.475-2.905$ & 0.728 \\
DAO & -0.066 & $0.594-1.477$ & 0.778 \\
\hline
\end{tabular}

$\mathrm{CP}$ classes in patients. In addition, we confirmed that the Firmicutes/Bacteroidetes ratio was positively correlated with the levels of above-mentioned bacteria. Additionally, alcohol consumption did not obviously altered the levels of these bacteria and serum parameters including D-lactate, endotoxin and DAO contents in patients with HBV-related liver cirrhosis.

The Firmicutes/Bacteroidetes ratio was reported to be associated with the morbidity and pathogenesis of several chronic diseases like obesity or diabetes [1315]. Obese population, both children and adults, had elevated Firmicutes/Bacteroidetes ratio compared with controls with normal weight $[13,16]$. On contrast, decreased Firmicutes/Bacteroidetes ratio in obese individuals was identified to be related with weight loss [17]. Cui et al. showed that the elevated the Firmicutes/ Bacteroidetes ratio in pigs was correlated with reduced fatty acid synthase (FASN) and acetyl-CoA carboxylase $\alpha(A C C \alpha)$ mRNA levels in liver, but opposed trends in subcutaneous fat. The inhibition of $A C C \alpha$ suppresses FASN and thus inhibits the synthesis of fatty acid from free fatty acids (FFAs) like saturated straight-chain FAs
[18]. Decreased FASN level was detected in adipose tissue from hypertensive individuals and subcutaneous adipose tissue from obese subjects [18-20]. What's more, FASN has a negative correlation with insulin resistance [21]. Consistently, our present study suggested that the increased Firmicutes/Bacteroidetes ratio was an independent risk facor for HBV-related liver cirrhosis, indicating the alterations in lipid metabolism and FAs biosynthesis in patients.

Megamonas and Veillonella genus were identified to be significantly contributed to the pathogenesis of liver disease. Both Megamonas and Veillonella genera are poorly characterized members of Veillonellaceae family, Firmicutes phylum. Veillonella genus is anaerobic genus [22]. However, both Megamonas and Veillonella are frequently elevated in cystic fibrosis cases [23], individuals with type I diabetes [22] and patients with primary sclerosing cholangitis with or without inflammatory bowel disease (IBD) compared with healthy controls [24, 25]. Veillonella genus is a producer of butyrate and shortchain fatty acids (SCFAs) [26] which contributes to antiinflammatory response in the host [27]. The elevated and decreased level of Megamonas was identified in the host with IBD [24] and Behcet's disease [28], respectively. Chakravarthy et al. detected the reduced Veillonella dispar level in patients with uveitis, an inflammatory disease of the eye [29]. By contrast, Matera et al. reported the increased level of Veillonella in the mouth flora in individuals with periodontal disease [30]. Additional in vitro evidence suggested that Veillonella LPS stimulated the release of cytokines like interleukin-6 (IL-6), IL-1 $\beta$, IL-10 and tumor necrosis factor alpha (TNF- $\alpha$ ) in human peripheral blood mononuclear cells [30]. These results suggested that intestinal Veillonella metabolites like LPS and Veillonella-modulated metabolites like SCFAs affect the liver pathology and inflammation in the host [9]. We found the level of Veillonella and Megamonas was positively and negatively correlated with Child-Pugh classes in patient with HBV-related liver cirrhosis, respectively. The elevated Veillonella genus and decreased Megamonas genus might suggest the unbalanced inflammatory status in patients with HBV-related liver cirrhosis compared with control.

Several observations suggested that alcohol consumption itself not only induces injury and inflammation in the intestine and liver but also causes dissimilarity in microbial community $[10,11]$. Our present study confirmed that there was no dissimilarity in microbial community between patients with and without alcohol consumption. But differences were observed in microbial community between healthy controls and patients with or without alcohol consumption. This suggested the influence of alcohol consumption on the pathogenesis of disease. 
Alcohol consumption increases intestinal permeability and therefore induces endotoxaemia [31, 32]. Some researchers showed the insignificantly influence of alcohol consumption on intestinal permeability and endotoxin [33]. Our correlation analysis showed that alcohol consumption was positively correlated with increased endotoxin level. Alcohol consumption upregulated endotoxin and D-lactate levels in patients with Child-Pugh class A disease, and DAO in patients with Child-Pugh class $C$ disease. However, alcohol consumption showed non-benefit to endotoxin in patients with Child-Pugh class $\mathrm{B}$ and $\mathrm{C}$ diseases. Further regression analysis showed alcohol consumption was not an risk factor for endotoxin and aforementioned intestinal bacteria in patients with HBV-related liver cirrhosis. The increased endotoxin, D-lactate and DAO in patients with different Child-Pugh classes by alcohol consumption might due to the increased intestinal permeability. These data might reveal that alcohol consumption played an important inducer role in the pathogenesis of but a faint function the progression of HBV-related liver cirrhosis.

\section{Conclusions}

Our present study demonstrated that the intestinal microbial community dissimilarity in patients with HBVrelated liver cirrhosis compared with healthy controls. Elevated Firmicutes/Bacteroidetes ratio, reduced Megamonas genus (Veillonellaceae family) level, increased Veillonella genus (Veillonellaceae family) level was associated with Child-Pugh classes in patients with HBVrelated liver cirrhosis. We additionally identified that alcohol consumption play important roles in the pathogenesis of HBV-related liver cirrhosis.

\section{Supplementary information}

Supplementary information accompanies this paper at https://doi. org/10.1186/s13099-019-0337-2.

Additional file 1: Figure S1. The relative abundance of communities at phylum level. CPA, CPB and CPC, patients with Child-Pugh class $A(n=30)$, $B(n=31)$ and C $(n=19)$ hepatosis (hepatitis B or hepatitis B virus-related cirrhosis).

\section{Abbreviations}

HBV: chronic hepatitis B virus; HCC: hepatocellular carcinoma; HCV: nonhepatitis C virus; NAFLD: non-alcoholic fatty liver disease; NASH: non-alcoholic steatohepatitis; CP: Child-Pugh; CPA: CP class A; CPB: CP class B; CPC: CP class C; DAO: diamine oxidase; OTUs: operational taxonomic units; FASN: fatty acid synthase; ACCa: acetyl-CoA carboxylase a; IBD: inflammatory bowel disease; TNF-a: tumor necrosis factor alpha.

\section{Acknowledgements}

Thank you to Xiang Yan from First Hospital of Lanzhou University for the guidance for this study. This work was supported by Scientific Research Program of Health Industry in Gansu Province (GSWSKY2017-40).

\section{Authors' contributions}

YDD and LQY designed the study, collected the samples, drafted the initial manuscript, reviewed and revised the manuscript. XBP, RRZh and CQM collected the biosamples and performed the DNA extraction. XBP, YDD, LQY, and CQM carried out the bioinformatic analysis. RRZh do some job in the manuscript revision. All authors read and approved the final manuscript.

\section{Funding}

This study was supported by Gansu Health Industry Research Program (GSWSKY2017-40).

\section{Availability of data and materials}

The datasets used and/or analysed during the current study are available from the corresponding author on reasonable request.

\section{Ethics approval and consent to participate}

Ethical approval and consent to participate (LDYYLL2018-142) was obtained from the ethical committees of First Hospital of Lanzhou University, Lanzhou, China.

\section{Consent for publication}

Not applicable.

\section{Competing interests}

The authors declare that they have no competing interests.

\section{Author details}

${ }_{1}^{1}$ Department of Infection, First Hospital of Lanzhou University, \#1 Donggang West Road, Lanzhou 730000, Gansu, China. ${ }^{2}$ First Clinical Medicine of Lanzhou University, Medical College of Lanzhou University, \#199 Donggang West Road, Lanzhou 730000, Gansu, China. ${ }^{3}$ Department of Laboratory, First Hospital of Lanzhou University, \#1 Donggang West Road, Lanzhou 730000, Gansu, China.

Received: 21 April 2019 Accepted: 28 October 2019

Published online: 26 November 2019

\section{References}

1. Aparna S, Johannes H, Mikolajczyk RT, et al. Estimations of worldwide prevalence of chronic hepatitis $B$ virus infection: a systematic review of data published between 1965 and 2013. Lancet. 2015;386(10003):154655. https://doi.org/10.1016/S0140-6736(15)61412-X.

2. Adaszko D, Sotelo J, Orlando M, et al. HIV, hepatitis B and C, syphilis and Tuberculosis prevalence in people deprived of liberty for criminal reasons in Argentina Final results of a national study. Int J Infect Dis. 2018;73:202. https://doi.org/10.1016/j.ijid.2018.04.3872.

3. Wen X, Su H, Wang Y, et al. Prevalence and natural course of occult hepatitis B virus infection in residents of two communities of Wuwei City, Gansu Province, China. JViral Hepatitis. 2018. https://doi.org/10.1111/ jvh.12805.

4. Kang Y, Cai Y. Gut microbiota and hepatitis-B-virus-induced chronic liver disease: implications for faecal microbiota transplantation therapy. J Hosp Infect. 2017;96(4):S0195670117301937. https://doi.org/10.1016/j. jhin.2017.04.007.

5. Loomba R, Seguritan V, LiW, et al. Gut microbiome-based metagenomic signature for non-invasive detection of advanced fibrosis in human nonalcoholic fatty liver disease. Cell Metab. 2017;25(5):1054-1062.e5. https:// doi.org/10.1016/j.cmet.2017.04.001

6. Jing $W$, Yang $W, X u Z$, et al. Gut microbial dysbiosis is associated with altered hepatic functions and serum metabolites in chronic hepatitis $B$ patients. Front Microbiol. 2017;8:02222. https://doi.org/10.3389/fmicb .2017.02222.

7. Yun Y, Chang Y, Kim HN, et al. Alterations of the gut microbiome in chronic hepatitis b virus infection associated with alanine aminotransferase level. J Clin Med. 2019;8(2):173. https://doi.org/10.3390/jcm80 20173.

8. Liu Q, Li F, Zhuang Y, et al. Alteration in gut microbiota associated with hepatitis $b$ and non-hepatitis virus related hepatocellular carcinoma. Gut Pathog. 2019. https://doi.org/10.1186/s13099-018-0281-6. 
9. Bashiardes S, Shapiro H, Rozin S, et al. Non-alcoholic fatty liver and the gut microbiota. Mol Metab. 2016;5(9):782-94. https://doi.org/10.1016/j. molmet.2016.06.003.

10. Mcc C, Lacerda NL, Ferreira CM, et al. Comparing the effects of acute alcohol consumption in germ-free and conventional mice: the role of the gut microbiota. BMC Microbiol. 2014;14(1):1-10. https://doi.org/10.1186/ s12866-014-0240-4

11. Stearns JC, Lynch MDJ, Senadheera DB, et al. Bacterial biogeography of the human digestive tract. Sci Rep. 2011;1(2840):170. https://doi. org/10.1038/srep00170.

12. Armougom F, Raoult D. Use of pyrosequencing and DNA barcodes to monitor variations in Firmicutes and Bacteroidetes communities in the gut microbiota of obese humans. BMC Genomics. 2008;9(1):576. https:// doi.org/10.1186/1471-2164-9-576.

13. Nagwa Al, Ragab SH, Abeer AE, et al. Frequency of Firmicutes and Bacteroidetes in gut microbiota in obese and normal weight Egyptian children and adults. Arch Med Sci. 2011;7(3):501-7. https://doi.org/10.5114/ aoms.2011.23418.

14. Cui C, Shen CJ, Jia G, et al. Effect of dietary Bacillus subtilis on proportion of Bacteroidetes and Firmicutes in swine intestine and lipid metabolism. Genet Mol Res. 2013;12(2):1766-76. https://doi.org/10.4238/2013. May.23.1.

15. Greenhill C. Gut microbiota: Firmicutes and Bacteroidetes involved in insulin resistance by mediating levels of glucagon-like peptide 1. Nat Rev Endocrinol. 2015;11(5):254. https://doi.org/10.1038/nrendo.2015.40.

16. Koliada A, Syzenko G, Moseiko V, et al. Association between body mass index and Firmicutes/Bacteroidetes ratio in an adult Ukrainian population. BMC Microbiol. 2017;17(1):120. https://doi.org/10.1186/s1286 6-017-1027-1.

17. Ley RE, Turnbaugh PJ, Samuel K, et al. Microbial ecology: human gut microbes associated with obesity. Nature. 2006;444(7122):1022-3. https ://doi.org/10.1038/4441022a.

18. Carrerasbadosa G, Pratspuig A, Puig T, et al. Circulating fatty acid synthase in pregnant women: relationship to blood pressure, maternal metabolism and newborn parameters. Sci Rep. 2016;6:24167. https://doi.org/10.1038/ srep24167.

19. Mayas MD, Ortega FJ, Macías-González M, et al. Inverse relation between FASN expression in human adipose tissue and the insulin resistance level. Nutr Metab. 2010;7(1):3. https://doi.org/10.1186/1743-7075-7-3.

20. Roberts R, Hodson L, Dennis AL, et al. Markers of de novo lipogenesis in adipose tissue: associations with small adipocytes and insulin sensitivity in humans. Diabetologia. 2009;52(5):882-90. https://doi.org/10.1007/ s00125-009-1300-4.

21. Gouri R, Resat U, Irina P, et al. The lipogenic enzymes DGAT1, FAS, and LPL in adipose tissue: effects of obesity, insulin resistance, and TZD treatment. J Lipid Res. 2006;47(11):2444. https://doi.org/10.1194/jlr.M600248-JLR200.

22. Vesth T, Ozen A, Andersen SC, et al. Veillonella, Firmicutes: microbes disguised as Gram negatives. Stand Genomic Sci. 2013;9(2):431-48. https ://doi.org/10.4056/sigs.2981345.
23. Murri M, Leiva I, Gomez-Zumaquero JM, et al. Gut microbiota in children with type 1 diabetes differs from that in healthy children: a case-control study. BMC Med. 2013;11(1):46. https://doi.org/10.1186/1741-7015-11-46.

24. Vaughn BP, Kaiser T, Staley C, et al. A pilot study of fecal bile acid and microbiota profiles in inflammatory bowel disease and primary sclerosing cholangitis. Clin Exp Gastroenterol. 2019;12(9):9-19. https://doi. org/10.2147/CEG.S186097.

25. Tunney MM, Field TR, Moriarty TF, et al. Detection of anaerobic bacteria in high numbers in sputum from patients with cystic fibrosis. Am J Respir Crit Care Med. 2008;177(9):995. https://doi.org/10.1164/rccm.20070 8-11510C.

26. Anand S, Kaur H, Mande SS. Comparative in silico analysis of butyrate production pathways in gut commensals and pathogens. Front Microbiol. 2016;7:01945. https://doi.org/10.3389/fmicb.2016.01945.

27. Richards JL, Yu AY, Mcleod KH, et al. Dietary metabolites and the gut microbiota: an alternative approach to control inflammatory and autoimmune diseases. Clin Transl Immunology. 2016;5(5):e82. https://doi. org/10.1038/cti.2016.29.

28. Shimizu J, Kubota T, Takada E, et al. Relative abundance of Megamonas hypermegale and Butyrivibrio species decreased in the intestine and its possible association with the T cell aberration by metabolite alteration in patients with Behcet's disease (210 characters). Clin Rheumatol. 2019. https://doi.org/10.1007/s10067-018-04419-8.

29. Chakravarthy SK, Jayasudha R, Prashanthi GS, et al. Dysbiosis in the gut bacterial microbiome of patients with uveitis, an inflammatory disease of the eye. Indian J Microbiol. 2018. https://doi.org/10.1007/s1208 8-018-0746-9.

30. Matera G, Muto V, Vinci M, et al. Receptor recognition of and immune intracellular pathways for Veillonella parvula lipopolysaccharide. Clin Vaccine Immunol. 2009;16(12):1804-9. https://doi.org/10.1128/CVI.00310-09.

31. Parlesak A, Schäfer $C$, Schütz T, et al. Increased intestinal permeability to macromolecules and endotoxemia in patients with chronic alcohol abuse in different stages of alcohol-induced liver disease. J Hepatol. 2000;32(5):742-7. https://doi.org/10.1016/S0168-8278(00)80242-1.

32. Rao RK, Seth A, Sheth P. Recent advances in alcoholic liver disease I. Role of intestinal permeability and endotoxemia in alcoholic liver disease. Am J Physiol Gastrointest Liver Physiol. 2004;286(6):881-4. https://doi. org/10.1152/ajpgi.00006.2004.

33. Wigg AJ, Roberts-Thomson IC, Dymock RB, et al. The role of small intestinal bacterial overgrowth, intestinal permeability, endotoxaemia, and tumour necrosis factor alpha in the pathogenesis of non-alcoholic steatohepatitis. Gut. 2001;48(2):206-11. https://doi.org/10.1136/gut.48.2.206.

\section{Publisher's Note}

Springer Nature remains neutral with regard to jurisdictional claims in published maps and institutional affiliations.

Ready to submit your research? Choose BMC and benefit from:

- fast, convenient online submission

- thorough peer review by experienced researchers in your field

- rapid publication on acceptance

- support for research data, including large and complex data types

- gold Open Access which fosters wider collaboration and increased citations

- maximum visibility for your research: over 100M website views per year

At BMC, research is always in progress.

Learn more biomedcentral.com/submissions 